\title{
Numerical Study of Duffing Nonlinearity in the Quantum Dot Embedded Nanomechanical Resonator
}

\author{
Yue Wang $\left(\mathbb{D},{ }^{1}\right.$ Ghulam Bary $\mathbb{D D}^{1}{ }^{1}$ Riaz Ahmad $\left(\mathbb{D},{ }^{1}\right.$ Dameng Yin, ${ }^{1}$ Shiwei Xie, ${ }^{1}$ Qing Lu, \\ Ilyas Khan $\mathbb{D}^{2},{ }^{2}$ Nawa Alshammari $\left(\mathbb{D},{ }^{3}\right.$ Nawaf $N$. Hamadneh $(\mathbb{D}){ }^{3}$ \\ and Mulugeta Andualemb $\mathbb{D}^{4}$ \\ ${ }^{1}$ Faculty of Science, Yibin University, Yibin 644000, China \\ ${ }^{2}$ Department of Mathematics, College of Science Al-Zulfi, Majmaah University, Al Majmaah 11952, Saudi Arabia \\ ${ }^{3}$ Department of Basic Sciences, College of Science and Theoretical Studies, Saudi Electronic University, \\ Riyadh 11673, Saudi Arabia \\ ${ }^{4}$ Department of Mathematics, Bonga University, Bonga, Ethiopia
}

Correspondence should be addressed to Yue Wang; wy6144@163.com, Ghulam Bary; ghulambary@gmail.com, and Mulugeta Andualemb; mulugetaandualem4@gmail.com

Received 26 September 2021; Accepted 9 November 2021; Published 24 November 2021

Academic Editor: Muhammad Shoaib Anwar

Copyright (c) 2021 Yue Wang et al. This is an open access article distributed under the Creative Commons Attribution License, which permits unrestricted use, distribution, and reproduction in any medium, provided the original work is properly cited.

\begin{abstract}
Geometry, electrostatics, and single-electron tunneling contribute to the nonlinearity in the quantum dot embedded nanomechanical resonator, while "Duffing term" is a kind of mathematics describing the third-order nonlinearity of the system as a whole. We study theoretically the influence of a variation of a mathematical parameter Fuffing term on the actual physical effect. The position probability distribution, the average current, and the displacement fluctuation spectrum with the different Duffing parameter and electromechanical coupling are obtained through numerically calculating the Fokker Planck equation. The mechanical bistability has been described by these quantities under different electromechanical coupling and Duffing parameters. We conclude that the nonlinearities of the nanotube resonator contribute to the mechanical bistability, which induces the asymmetry of the position probability distribution, compresses the current, and softens or stiffens the mechanical resonance frequency as the same as the electromechanical coupling to use it in mechanical engineering.
\end{abstract}

\section{Introduction}

The linear dynamic description of nanoelectromechanical systems (NEMS) is well understood when the electromechanical coupling is rather weak [1-9]; meanwhile, the existence of the nonlinearities induces too many interesting consequences such as the nonlinear dynamic response in the two-dimensional material membranes, the mechanical bistability in the carbon nanotube (CNT)-based resonator, [10-13], and unusual mechanical response $[8,14-16]$. The CNT quantum dot embedded resonators have been widely investigated as ultrasensitive detectors and sensors [17-19]; the experimental research group reported higher record sensitivity in mass and force sensing [20, 21]. Recently, people have found that strong coupling induces strong nonlinear signals in the mechanical resonance frequency and the mechanical noise in the single- or two-level quantum nanomechanical oscillator [22-24]. The strong coupling dominates the quadratic term in the restore force than the geometry, electrostatics, and single-electron tunneling. The nonlinear response of the system contains a mechanical resonator at nanoscale coupled to a transistor of single electron under the external drive which behaves like the Duffing oscillator [25].

The intrinsic nonlinearities of the carbon nanotube have been ignored by most of these research studies. For the given carbon nanotube-based resonators, the nonlinearities come not only from the electromechanical coupling but also from the parent materials themselves. It is significant to consider the intrinsic nonlinearities of the carbon nanotube. In this 
work, we pay attention to the term of the Duffing under the force of the restoring with the cubic structure of the considered displacement. We are also interested in the nonlinear CNT-based resonators which will be described by the position probability distribution, the current, and the displacement fluctuations with the different coupling and Duffing parameter [26].

In particular, nonlinearities examine the quantum coherence pure substance which is a pure state of matter that can be quite sensitive. When two identical particles do not overlap, they each have their perfect quantum system described by the quantum oscillator. However, the measured nonlinearities may have been in a miniature form or even stay purest after the quantum mechanical overlapping. It is related to the strength, density, and temperature of the considered system (quantum entanglement). During transition times, when the density concentration is high, bouncing may potentially play a role in quantum waveparticle duality. Hydrodynamics is frequently used to characterize the extremely dense medium formed, which implies local thermodynamic equilibrium and quantum decoherence well above thermal length scale [27].

In this curious and unique research work, we probe the nonlinearities, consequences of average current flow, and intrinsic fluctuations of the probability distributions, as well as the suppression of the displacement under the properties of the considered pioneer quantum system with nanotubes. The creation of a useable source which inflates relativistic interior of the considered field potential about the special oscillator that evaluates over time in the phase of quantum temperature regimes, and we also compute the various parameters for numerous order quantum characteristics by applying the one and two nanotubes for the mentioned phases at distinct temperature and momenta regimes. The nonlinearity of production systems influences the critical parameter significantly under the considered measurements at miscellaneous temperatures' dependence noise which are explored to use in thermal and mechanical applications of engineering.

The study is organized as follows. In Section 2, we introduce our model with the description of the system which consists of the carbon nanotube quantum dot embedded resonator; then, the numerical method is introduced, the Langevin equation are given, and the Fokker Planck equation is discretized to obtain the stationary solution. In Section 3, we study three physical quantities such as the position probability distribution, the average current, and the displacement fluctuations spectrum with the presence of nonlinearity of the CNT and different electromechanical couplings. The mechanical bistability has been described by them. Finally, in Section 4, we present our conclusions.

\section{Methods}

The Hamiltonian is introduced by William Rowan Hamilton, who created a ground breaking reconceptualization of Newtonian mechanics called as Hamiltonian dynamics, which was substantially vital to the future of quantum physics. Therefore, the Hamiltonian of the quantum dot embedded CNT mechanical resonator (see Figure 1).
We obtain

$$
H=H_{r e}+H_{Q}+H_{c},
$$

where the first term denotes the single-mode mechanical resonator. Specifically, in this study, we consider the influences of the force of restoring under the Duffing term which is cubic within displacement $x$. The restoring force is illustrated as

$$
F_{r}=-k x-\alpha x^{3},
$$

where $\alpha$ is the Duffing parameter and then the first term reads:

$$
H_{r e}=\frac{p^{2}}{2 m}+\frac{m \omega^{2}}{2} x^{2}+\alpha x^{4},
$$

where the considered mass $m$, momentum $p$, and especially the resonating frequency which possess the inverse relation with the mass can be illustrated as $\omega^{2}=(\mathrm{k} / \mathrm{m})$. In the second term, the Hamiltonian is composed of three parts; two of them are with the specific energy of the quantum dot and electrons, respectively. We consider the CNT as a single-level quantum dot mentioned in [30] with the width of the single level with the dependence of density of the considered states. Such mathematical representations can be manipulated as $\Gamma=\Gamma_{l}+\Gamma_{r}$, and the last one is the energy of tunneling [28]. The detail expression of the second term is written as $H_{Q}=\sum_{\alpha, k}\left(\varepsilon_{\alpha k}-\mu_{\alpha}\right) c_{\alpha k}^{\dagger} c_{\alpha k}+\epsilon_{0} d^{\dagger} d+\sum_{k} t_{\alpha k} c_{\alpha k}^{\dagger} d+H \cdot c$, which possess the factor $\alpha=L(r)$, and $\varepsilon_{\alpha k}$ determines the electrons energy summation in the exaggeration and the energy of chemical potential is $\mu_{\alpha}$. However, the terms $d$ as well as $c$ represent the dot and destruction operator, respectively. When the tunnel of electron appears at the CNTs, the corresponding rate of tunneling can be mentioned as

$$
\Gamma_{\alpha}=\pi t_{\alpha}^{2} \rho_{\alpha},
$$

where $\rho_{\alpha}$ represents the density of the considered states. We consider the symmetric system to simplify our findings, and in such techniques, we have to write $\Gamma / 2=\Gamma_{l}=\Gamma_{r}$.

The last term that is more crucial and interesting in the nanomechanical systems gives the coupling strength of electromechanical for the aforementioned system, and one can read it as [30]

$$
H_{c}=-F_{0} x d^{\dagger} d .
$$

One could find that the coupling strength is the function of the vibration displacement within the CNTs. In such quantum calculations, the factor $n=d^{\dagger} d$ denotes the numbering of electrons at the average under the tunneling in $\mathrm{CNTs}$, and $F_{0}$ represents the active force for the oscillator at that moment when an electron is tunneling just at the top of the CNTs.

In particular, we consider the electron tunnel in the coherent regime under the condition $k_{B} T \ll \Gamma$. In such circumstances, the factor $\Gamma$ represents the rate of tunneling, and $T$ measures the strength of the considered temperature. Especially, $\hbar$ as well as $k_{B}$ denote the Planck and the Boltzmann important constants, respectively. In particular, 


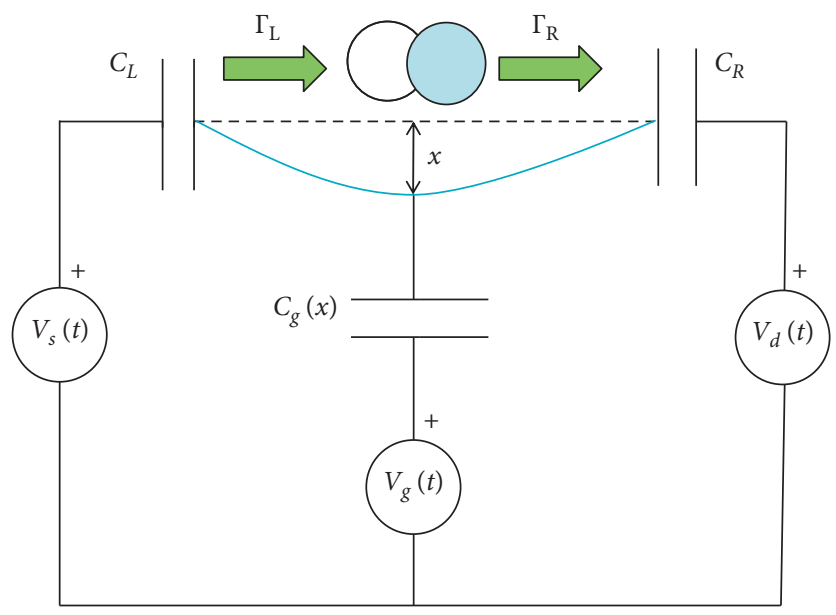

FIGURE 1: Schematics of the quantum dot embedded nanomechanical resonator. The mentioned CNT is clamped doubly between a drain electrode and source which has been suspended just above the given gate electrode; then, the CNT acts as a quantum dot, and single-electron tunneling is the dominant transport mechanism.

the mechanical mode dynamics with the displacement can be illustrated by a famous equation of Langevin [14]:

$$
m \ddot{x}+A(x) \dot{x}+m \omega_{0}^{2} x=e t a(t)+F_{e}(x),
$$

where $A(x)$ represents the dissipation and $\eta(t)$ is a force of stochastic that obeys the $\left\langle\eta(t) \eta\left(t^{\prime}\right)\right\rangle=C(x) \delta\left(t-t^{\prime}\right)$. Because the effect of the time interval of the correlations functions can denote $\Gamma^{-1}$, in such circumstances, the acquired $\Gamma \gg \omega_{0}$. So, we approximate the considered calculations by using the function of Dirac delta and the force at an average manipulates as $F_{e}(x)=F_{0} n_{d}(x)$ and $C(x)=F_{0}^{2} S_{n n}(x, \omega \sim 0)$, and similarly, the expression of $A(x)=-\left.F_{0}^{2}\left(\partial S_{n n} / \partial \omega\right)(x, \omega)\right|_{\omega=0}$, within the limit of $n_{d}=\left\langle d^{\dagger} d\right\rangle$ which examines the mean of the population of our considered dot. $m$ also measures the effectiveness of the mass for the oscillator mode considered. The obvious expressions for $n_{d}, S_{n n}$, and $A$ have been derived in detail in the Appendix of [15]. With the equation of Langevin, one can obtain the probability distributions $Q(x, p, t)$ within the framework of the Fokker Planck form:

$$
\partial_{t} Q=\frac{p}{m} \partial_{x} Q-F \partial_{p} Q+\frac{A}{m} \partial_{p}(p Q)+\frac{D}{2} \partial_{p}^{2}(Q)
$$

where $F(x)=F_{r}+F_{e}(x)$.

\section{Results and Discussion}

The main object of this research is to explore the nature of the nanomechanical system which is originated by quantum mechanical systems at an extraordinary momentum, and it can be feasible by using the system of carbon nanotubes' resonator with numerous cavities and their corresponding mechanical restoring force consistently. Here, we elucidate our model results about nonlinearities of the system, position probability distribution, average current, and displacement fluctuations. We are also interested to examine the characteristics of the position probability, mechanical noise, and average current which behaved meaningful significantly during the considered quantum systems at various energies and momenta, and we presume that the produced sources consist of the debris of frequencies that behave as the nanomechanical system to use in the thermal as well as in mechanical engineering.

3.1. Nonlinearities of the System. In this section, we introduce nonlinearity in our study; the nonlinearity can have various sources. Here, we are interested to explore in the famous and unique sources which are the two within the main unit. One is directly from the electron tunneling, which contributes the $F_{e}$ in the total force and exhibits from the electromechanical coupling $\alpha_{S E T}$. Another source is from the geometry $\alpha_{G}$ and electrostatic $\alpha_{E S}$ indirectly. In recent CNT resonator-based experiments [27], one can estimate the Duffing parameter $\alpha=-1 /(6 m) \mathrm{d}^{3} F / \mathrm{d} x^{3}$, when $V_{g}=1 V, \alpha_{S E T}=8 * 10^{31} \mathrm{~N}$ $/\left(\mathrm{kg} * \mathrm{~m}^{3}\right), \alpha_{G}=9.6 * 10^{34} \mathrm{~N} /\left(\mathrm{kg} * \mathrm{~m}^{3}\right)$, and $\alpha_{E S}=$

$-3.2 * 10^{28} \mathrm{~N} /\left(\mathrm{kg} * \mathrm{~m}^{3}\right)$. One can write the Duffing term in the restoring force as the nonlinearities. Now, the total resultant force $F(x)$ exerts at the considered oscillator, and such force can be seen in [30]. We also here considered that the vanishing temperature factor which implies $k_{B} T \ll \Gamma$, as well as the lowest voltages which are bias and demonstrate $e V \ll \Gamma$, could be acquired with the summation of the force restoring factor within the mechanical system and the electronic contribution as mentioned in equation (2). The force due to the electronic contribution refers to [15] and can be illustrated as

$$
F_{e}(x)=f\left(x, \varepsilon_{0}, \varepsilon_{P}\right) .
$$

One could see that the Duffing parameter $\alpha$, the variation of the gate energy $\varepsilon_{0}$, and coupling $\varepsilon_{P}$ could contribute the nonlinearities for the system.

3.2. Position Probability Distribution. In this section, we discuss the mechanical motion by the position probability distribution of the CNT with the Duffing parameter and the 
electromechanical coupling. In order to solve the considered equation of Fokker Planck, we examine and investigate the given equation to solve sophistically and numerically within the linear problem. Defining the operator of the famous Fokker Planck as mentioned $\mathscr{L}$ in the acquired equation,

$$
\partial_{t} Q=\tilde{\mathscr{L}} Q
$$

For the stationary solution, $\partial_{t} Q=0$, we consider the oscillator of quantum mechanical systems in the presence of the symmetry, where the gate energy $\varepsilon_{0}=\varepsilon_{P} / 2$ shows the special characteristics, and it also corresponds to adopting the most new point of electron hole symmetry for the aforementioned system [15]. Moreover, the parameters $\omega_{0} / \Gamma=10^{-3}$ and $k_{B} T / \Gamma=10^{-2}$ also can be useful in the measured calculation. The units of the $t, x, p$, and $\alpha$ are $\omega_{0}^{-1}$, $\left(m \omega_{0}\right)^{-1 / 2},\left(m \omega_{0}\right)^{1 / 2}$, and $F_{0}\left(m \omega_{0}\right)^{3 / 2}$ in this paper. From Figure 2, the mechanical bistability of this system has been described when Duffing parameter, $\alpha=0$; these results work well with the conclusions of [15]. We discuss our results from the mechanical view when the Duffing parameter $\alpha=0$; the total force is

$$
F(\tilde{x})=-k \tilde{x}+\frac{F_{0}}{\pi} \arctan \frac{F_{0} \tilde{x}}{\Gamma} .
$$

where $\tilde{x}=x-x_{0}$ and $x_{0}=F_{0} / 2 k$. One can verify that the acquiring force is not symmetric corresponding to the acquiring points of $x_{0}=F_{0} / 2 k$ and linear with $\tilde{x}$. The position probability distribution shows that the coupling increases over the critical coupling value $\varepsilon_{c}=\pi \Gamma$. The position probability distribution displays a strong nonlinear signal which is that merely one curious sharp-peak turns to double peaks which show that the coupling induces strong nonlinear effect and the considered system tilts into the regimes of the bistable $\widetilde{\varepsilon}_{p}>1$ which originate from basic single stable regime $\varepsilon_{p} \leq \varepsilon_{c}$. For the critical value, our system distribution possess flat and wider sharp peaks. All these interesting consequences could be explained by the potential of the system. One could see that the nonlinear effect from the coupling is dominant in the tunneling process than the Duffing nonlinearity. The position probability distribution with different Duffing parameters shows that the nonlinearity of the nanotube resonator also contributes to the mechanical state's transition; it induces the asymmetric of the position probability distribution with $x_{0}$. It could push or slow down the process of electron tunneling.

3.3. The Average Current. Because of its mobility, a quantum particle like an electron generates an electric current. The flow of its likelihood is linked to that current. These currents are determined by the wave function that explains a particle's state. At a higher level, the quantum operator that can operate between states or interact with a density matrix to determine currents even in scenarios such mixed levels of thermodynamic equilibrium which can be found. Here are the concepts and equations which were used to utilize these themes. The current in our model under the coherent tunneling regime shows $k_{B} T \ll \Gamma$ and under the certain condition implies $V, T \ll \Gamma[29]$. In such circumstances, the limit of the lower voltage obeys the condition of $e V \ll k_{B} T$, and it can manipulate as [30]

$$
I=\frac{e^{2} V}{2 \pi} \tau(z),
$$

where $\tau=1 /\left(1+z^{2}\right)$ and $z=\left(\mu-\epsilon_{0}+F_{0} x\right) / \Gamma$. It is easier to commute the average of the current $\omega \ll \Gamma$ with the distribution of the probability $P_{s t}$ by integrating $x$ :

$$
I=\frac{e^{2} V}{2 \pi} \int \mathrm{d} x \mathrm{~d} p Q(x, p, t) \tau(x) .
$$

From Figure 3, one could see that the strong electromechanical coupling strongly suppresses the current, which is called the current blockade. The presence of the Duffing nonlinearities could accelerate or slow down the current's declining, especially in the region of the bistable state.

3.4. Displacement Fluctuations. In this section, we investigate the effect of the electromechanical coupling and the Duffing nonlinearity on the system from the displacement fluctuations spectrum. The displacement fluctuations' spectrum is as [13]

$$
S_{x x}(\omega)=-2 \operatorname{Tr}\left[\widehat{\widetilde{x}} \frac{\hat{\mathscr{L}}}{\omega^{2}+\hat{\mathscr{L}}^{2}} \widehat{\widetilde{x}} Q_{s t}\right] .
$$

Here, it is crucial to indicate that the whole terms which acquired a hat in (13) represent the super quantum operators those acting in the regime of the probability space and can be illustrated as $\widehat{\widetilde{x}}(t)=\widehat{x}(t)-\langle x\rangle$.

From Figure 4, one could see that, in the mechanical frequency $\omega_{0}$ region when the system contains only monostable state, there is appearing unit resonance frequency with the sharpness peak. Once the considering coupling increases over the certain value, the mentioned sharp-peak transference to the extremely low frequency and the corresponding width of mentioned peak becomes broaden and broaden with the softening of an interesting frequency which represents the mechanical phenomena. The Duffing nonlinearity can contribute to the resonance frequency shift under the specific condition that the considered system goes to that regime which is bistable. However, the appearance of the doublet peak originates as the electrons can transform from one identical state to distinct another state. Therefore, the identical fluctuation of displacement spectrum features has also been obtained in [15] and [22]. Both situations appear for a system of nanomechanical within the regime of the semiclassical and the Frank-Condon principle [11]. When the Duffing term $\alpha$ is negative, the resonance frequency shifts to a higher frequency, meaning the stiffening spring behavior. Conversely, within the limit of $\alpha$ which took positive and the corresponding resonance certain peak shifts towards the critical lower frequency, meaning the softening-spring behavior. 


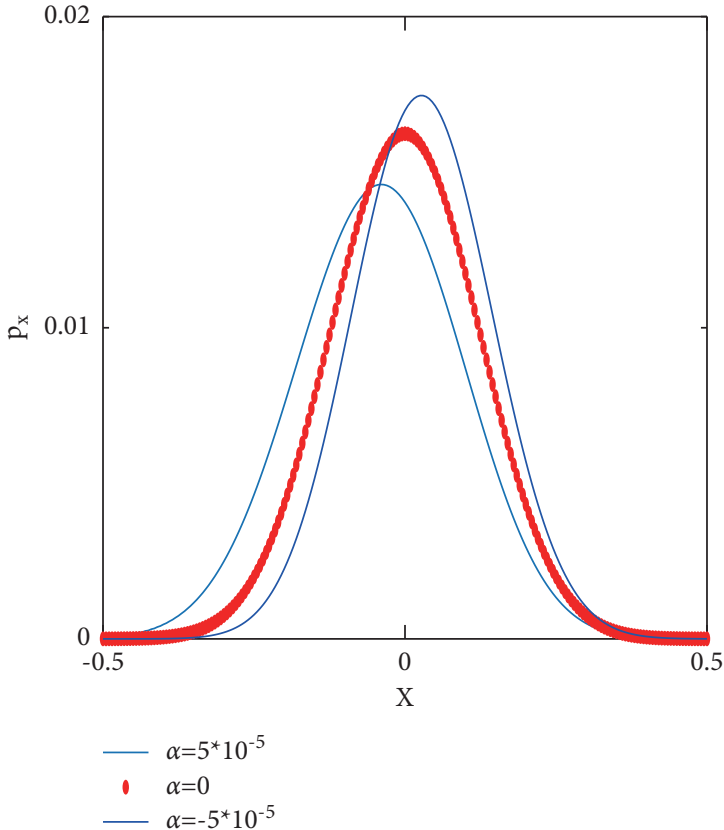

(a)

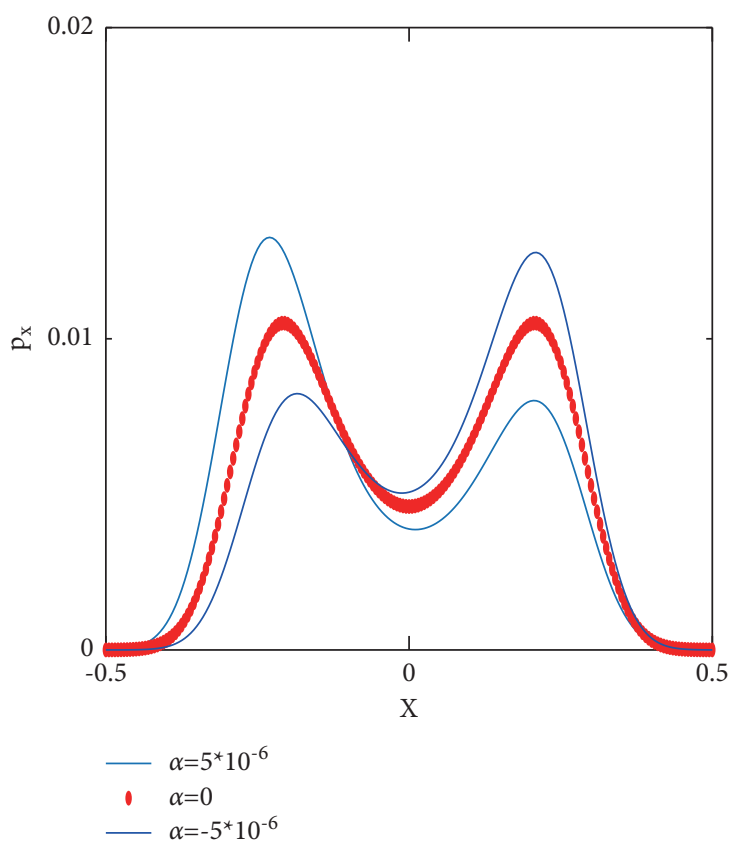

(b)

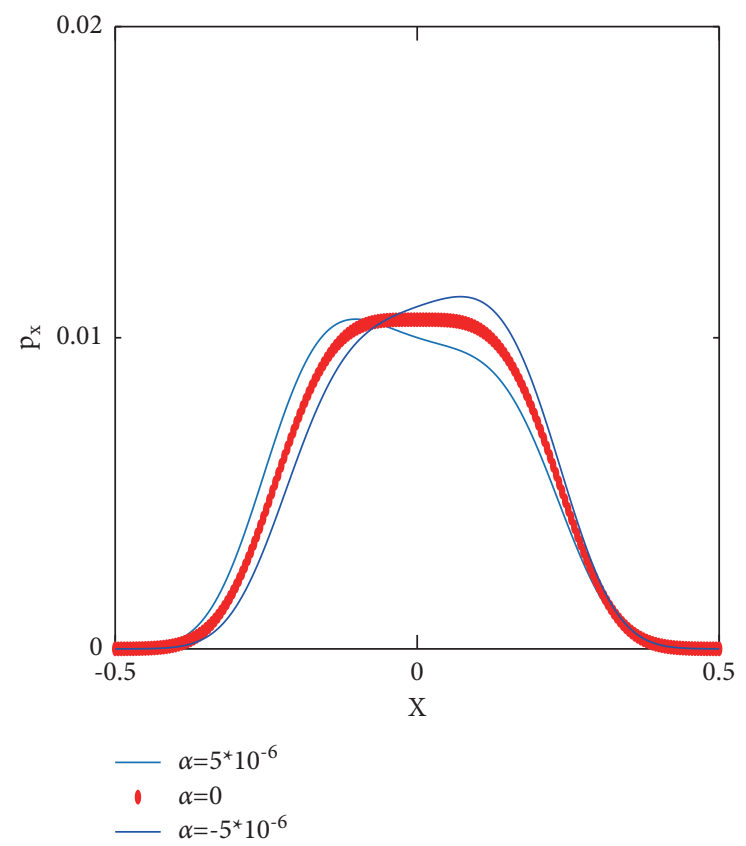

(c)

Figure 2: The position probability distribution $P_{x}$ versus displacement with different coupling $\left(\varepsilon_{P}=0.6 \pi \Gamma, 1 \pi \Gamma, 1.2 \pi \Gamma\right)$ and three Duffing parameters $\left(\alpha=-5 * 10^{-5}, 0,5 * 10^{-5}\right)$. 


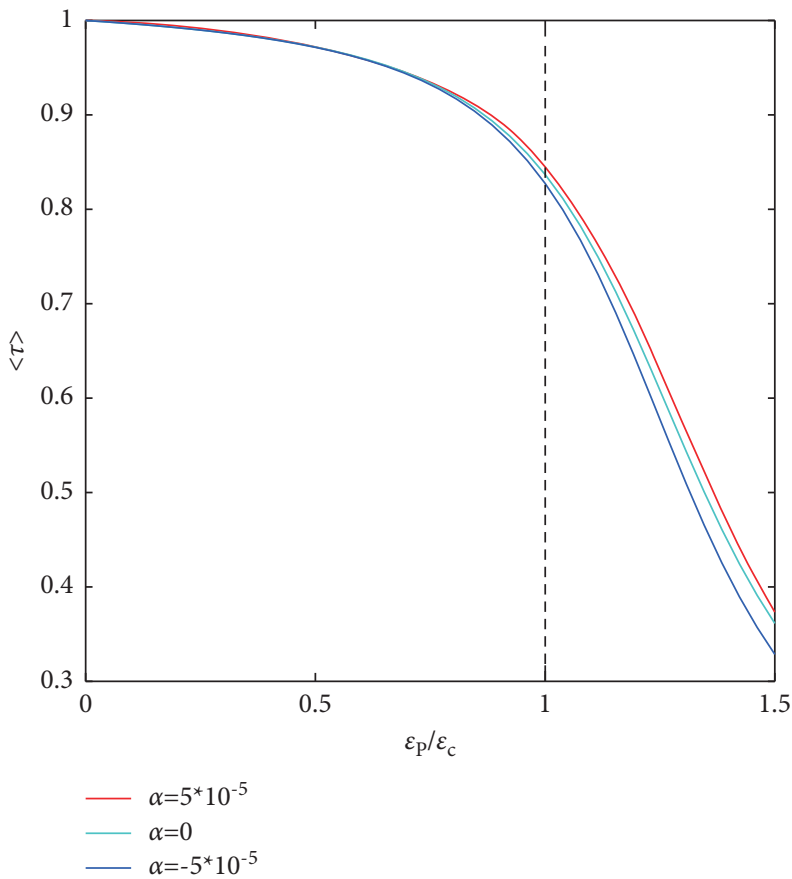

FIgURE 3: The average current $\langle\tau\rangle$ versus the electromechanical coupling with three Duffing parameters $\left(\alpha=-5 * 10^{-5}, 0,5 * 10^{-5}\right)$.
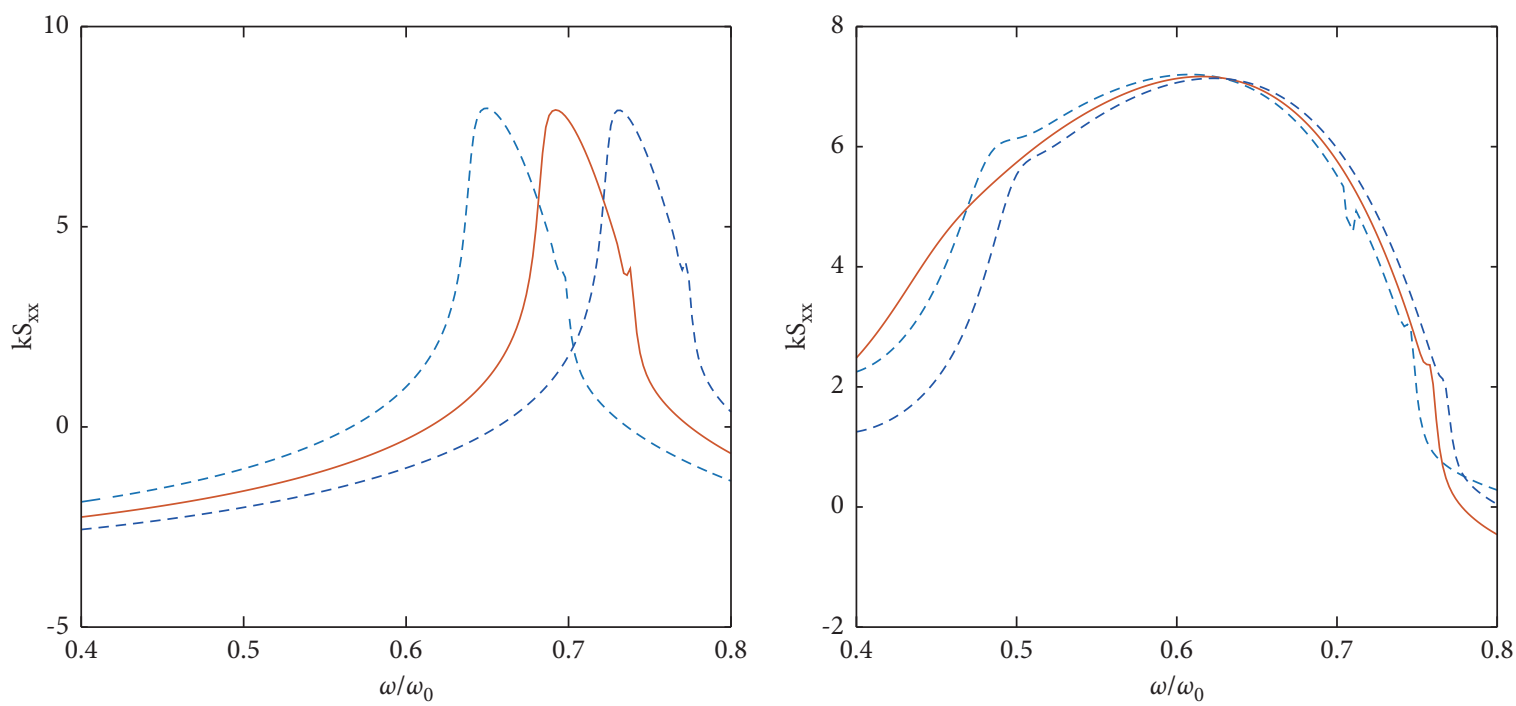

$$
\begin{aligned}
& -\alpha=5^{\star} 10^{-5} \\
& -\alpha=0 \\
& --\alpha=-5^{\star} 10^{-5}
\end{aligned}
$$$$
\begin{aligned}
& -\alpha=5^{\star} 10^{-5} \\
& -\alpha=0 \\
& --\alpha=-5^{\star} 10^{-5}
\end{aligned}
$$

(a)

(b)

Figure 4: Continued. 


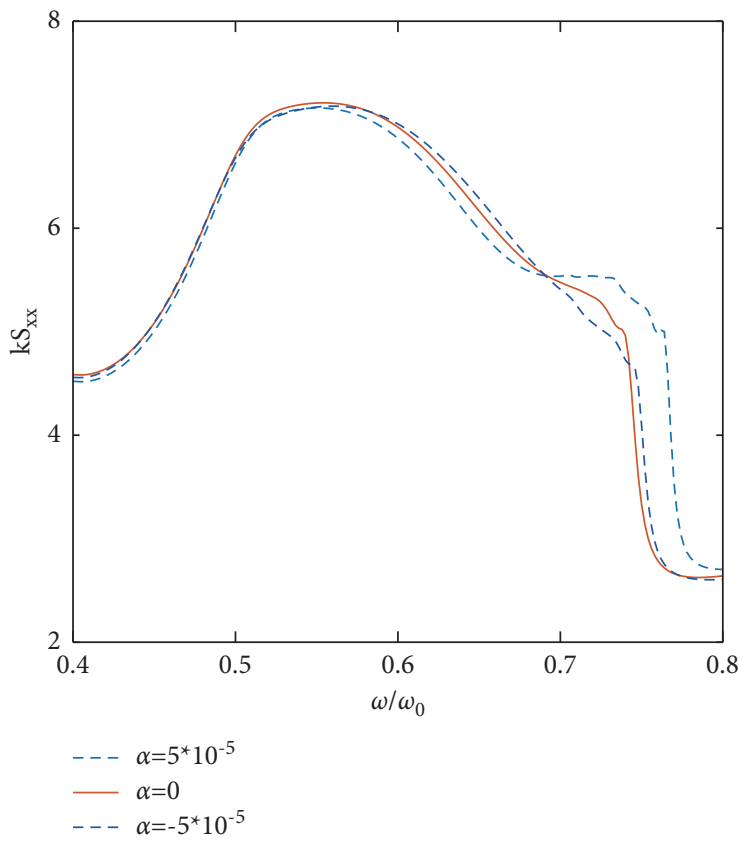

(c)

Figure 4: The dimensionless fluctuations of displacement spectrum versus the mechanical distribution of frequency within the various coupling strength $\left(\epsilon_{P}=0.60 \pi \Gamma, \pi \Gamma, 1.20 \pi \Gamma\right)$ and the three Duffing parameter $\left(\alpha=-5 * 10^{-5}, 0,5 * 10^{-5}\right)$.

\section{Conclusions}

We afford the research of the nonlinearities in the nanomechanical system of a carbon nanotube resonator strongly coupled to a quantum dot. The two main sources of the nonlinearities are given from the mechanical restoring force; one comes from directly the single-electron tunneling, especially with the presence of the strong electromechanical coupling; another nonlinearity is from geometry, electrostatics, and electron tunneling. The Duffing term is considered that is cubic in displacement included in the restoring force in our paper. The position probability, the average current, and the mechanical noise with different Duffing parameters have been studied. It is found that the Duffing nonlinearities of the CNT-based resonator could induce the asymmetry of the probability distribution of the current blockade, soften, or stiffen the mechanical resonance frequency that is important as the electromechanical coupling in the nanomechanical systems.

We have explored the nonlinearities of the system, position probability distribution, average current, and displacement fluctuations. We have also investigated the characteristics of the position probability, mechanical noise, and average current with various factors which behaved meaningful during the considered quantum systems at distinct energies, momenta in the short, and wide temperature regimes. One persuasive explication of this ramification is that our considered quantum system possessed the asymmetry of the probability distribution.

\section{Data Availability}

The data used to support the findings of the study are available within the article.

\section{Disclosure}

This paper is new and not submitted to any other journal. All the results included are new and not reported before.

\section{Conflicts of Interest}

The authors declare that they have no conflicts of interest or personal relationships that could have appeared to influence the work reported in this paper.

\section{Authors' Contributions}

Yue Wang put forward the ideal and wrote the original draft; Ghulam Bary and Riaz Ahmad wrote the manuscript; Dameng Yin and Shiwei Xie performed the calculations; Qing Lu, Ilyas Khan, Nawa Alshammari, Nawaf $\mathrm{N}$. Hamadneh, and Mulugeta Andualemb contributed the check and review of the results and data analysis.

\section{Acknowledgments}

This work was supported by the Sailing Plan Project of Yibin University (nos. 2021QH08, 2021QH05, and 2021QH06) and the Open Research Fund of Computational physics Key 
Laboratory of Sichuan province, Yibin University (nos. YBXYJSWL-ZD-2020-003 and YBXYJSWL-ZD-2020-004). The authors thank Xulin Peng and Lin Li for careful read and useful suggestions. Yibin University provided the financial support for this study.

\section{References}

[1] C. Dutreix, R. Avriller, and B. Lounisand, "Two-level system as topological actuator for nanomechanical modes," Physical Review Research, vol. 2, Article ID 023268, 2020.

[2] Q. Schaeverbeke, R. Avriller, T. Frederiksen, and F. Pistolesi, "Single-photon emission mediated by single-electron tunneling in plasmonic nanojunctions," Physical Review Letters, vol. 123, no. 24, Article ID 246601, 2019.

[3] R. Avriller, B. Murr, and F. Pistolesi, "Bistability and displacement fluctuations in a quantum nanomechanical oscillator," Physical Review B, vol. 97, no. 15, Article ID 155414, 2018.

[4] Y. M. Blanter, O. Usmani, and a. Y. V. Nazarov, "Singleelectron tunneling with strong mechanical feedback," Physical Review Letters, vol. 93, no. 13, Article ID 136802, 2004.

[5] Ya. M. Blanter, O. Usmani, and E. Nazarov, "Single electron tunneling with strong mechanical feedback," Physical Review Letters, vol. 94, Article ID 049904, 2005.

[6] A. D. Armour, M. P. Blencowe, and Y. Zhang, "Classical dynamics of a nanomechanical resonator coupled to a singleelectron transistor," Physical Review B, vol. 69, no. 12, Article ID 125313, 2004.

[7] C. B. Doiron, W. Belzig, and C. Bruder, "Electrical transport through a single-electron transistor strongly coupled to an oscillator," Physical Review B, vol. 74, no. 20, Article ID 205336, 2006.

[8] F. Pistolesi and R. Shekhter, "Tunable spin-polaron state in a singly clamped semiconducting carbon nanotube," Physical Review B: Condensed Matter, vol. 92, Article ID 035423, 2015.

[9] R. Ahmad, A. Farooqi, and R. Farooqi, "An analytical approach to study the blood flow over a nonlinear tapering stenosed artery in flow of carreau fluid model measles," Complexity, vol. 2021, Article ID 9921642, 11 pages, 2021.

[10] D. Mozyrsky, M. B. Hastings, and I. Martin, "Intermittent polaron dynamics: born-Oppenheimer approximation out of equilibrium," Physical Review B: Condensed Matter, vol. 73, Article ID 035104, 2006.

[11] J. Koch and F. von Oppen, "Franck-condon blockade and giant fano factors in transport through single molecules," Physical Review Letters, vol. 94, no. 20, Article ID 206804, 2005.

[12] F. Pistolesi and S. Labarthe, "Current blockade in classical single-electron nanomechanical resonator," Physical Review $B$, vol. 76, no. 16, Article ID 165317, 2007.

[13] F. Pistolesi, Y. M. Blanter, and I. Martin, "Self-consistent theory of molecular switching," Physical Review B: Condensed Matter, vol. 78, Article ID 085127, 2008.

[14] G. Micchi, R. Avriller, and F. Pistolesi, "Mechanical signatures of the current blockade instability in suspended carbon nanotubes," Physical Review Letters, vol. 115, no. 20, Article ID 206802, 2015.

[15] G. Micchi, R. Avriller, and F. Pistolesi, "Electromechanical transition in quantum dots," Physical Review B, vol. 94, no. 12, Article ID 125417, 2016.

[16] J. Moser, A. Eichler, J. Güttinger, M. I. Dykman, and A. Bachtold, "Nanotube mechanical resonators with quality factors of up to 5 million," Nature Nanotechnology, vol. 9, no. 12, pp. 1007-1011, 2014.

[17] A. Benyamini, A. Hamo, S. V. Kusminskiy, F. von Oppen, and S. Ilani, "Real-space tailoring of the electron-phonon coupling in ultraclean nanotube mechanical resonators," Nature Physics, vol. 10, no. 2, pp. 151-156, 2014.

[18] M. Ganzhorn and W. Wernsdorfer, "Dynamics and dissipation induced by single-electron tunneling in carbon nanotube nanoelectromechanical systems," Physical Review Letters, vol. 108, no. 17, Article ID 175502, 2012.

[19] K. L. Ekinci, X. M. H. Huang, and M. L. Roukes, "Ultrasensitive nanoelectromechanical mass detection," Applied Physics Letters, vol. 84, no. 22, pp. 4469-4471, 2004.

[20] J. Moser, J. Güttinger, A. Eichler et al., "Ultrasensitive force detection with a nanotube mechanical resonator," Nature Nanotechnology, vol. 8, no. 7, pp. 493-496, 2013.

[21] B. Lassagne, D. Garcia-Sanchez, A. Aguasca, and A. Bachtold, "Ultrasensitive mass sensing with a nanotube electromechanical resonator," Nano Letters, vol. 8, no. 11, pp. 3735-3738, 2008.

[22] R. Avriller, B. Murr, and F. Pistolesi, "Bistability and displacement fluctuations in a quantum nanomechanical oscillator," Physical Review B, vol. 97, no. 15, Article ID 155414, 2018.

[23] F. Pistolesi, "Bistability of a slow mechanical oscillator coupled to a laser-driven two-level system," Physical Review A, vol. 97, Article ID 063833, 2018.

[24] V. Puller, B. Lounis, and F. Pistolesi, "Single molecule detection of nanomechanical motion," Physical Review Letters, vol. 110, no. 12, Article ID 125501, 2013.

[25] P. G. Kirton and A. D. Armour, "Nonlinear dynamics of a driven nanomechanical single-electron transistor," Physical Review B, vol. 87, no. 15, Article ID 155407, 2013.

[26] A. A. Clerk, "Quantum-limited position detection and amplification: a linear response perspective," Physical Review B, vol. 70, no. 24, Article ID 245306, 2004.

[27] G. Bary, W. Ahmed, M. Sajid et al., "A new analytical approach to study chaos fraction characterization by using intensity interferometry," Chaos, Solitons \& Fractals, vol. 152, Article ID 111414, 2021.

[28] Y. Wang, F. Liu, and D. Huang, "Electromechanical coupling effect in the detection of nanomechanical motion," The European Physical Journal B, vol. 94, no. 5, p. 107, 2021.

[29] Y. M. Blanter and M. Büttiker, "Shot noise in mesoscopic conductors," Physics Reports, vol. 336, no. 1-2, pp. 1-166, 2000.

[30] Y. Wang, G. Micchi, and F. Pistolesi, "Sensitivity of the mixing-current technique for the detection of mechanical motion in the coherent tunnelling regime," Journal of Physics: Condensed Matter, vol. 29, no. 46, Article ID 465304, 2017. 